\title{
CORRIGENDA
}

Estimation of body composition of sheep by isotopic dilution techniques.

\section{Exchangeable potassium}

By E. A. Domingo, T. E. Trigg and J. H. Topps

Volume 32, no. I

Page 20A, Table I :

$$
\begin{gathered}
\text { For FFEBW }=0.419 \mathrm{~K}_{\mathrm{e}}-0.55 \mathrm{I} \\
\text { Read FFEBW }=0.4 \mathrm{I} 9 \mathrm{~K}_{\mathrm{e}}-0.59 \mathrm{I} \\
\text { For } \mathrm{EBN}=0.012 \mathrm{~K}_{\mathrm{e}}+0.00 \mathrm{I} \mathrm{W}+0.07 \\
\text { Read } \mathrm{EBN}=0.012 \mathrm{~K}_{\mathrm{e}}+0.00 \mathrm{I} \mathrm{W}+0.007 \\
\text { For } \mathrm{FFEBW}=0.276 \mathrm{~K}_{\mathrm{e}}+0.27 \mathrm{IW}-0.0 \mathrm{I} 5 \\
\text { Read } \mathrm{FFEBW}=0.276 \mathrm{~K}_{\mathrm{e}}+0.27 \mathrm{IW}-\mathrm{I} .5
\end{gathered}
$$

Estimation of body composition of sheep by isotopic dilution techniques.

1. Deuterium oxide and tritiated water

By T. E. Trigg, E. A. Domingo and J. H. Topps

Volume 32, no. I

Page 2i A, Table I :

$$
\begin{aligned}
& \text { For } \mathrm{EBF}=0.406 \mathrm{~W}+0.552 \mathrm{D}+0.2 \mathrm{II} \\
& \text { Read } \mathrm{TBF}=0.406 \mathrm{~W}-0.552 \mathrm{D}+2 . \mathrm{I} \mathrm{I} \\
& \text { For } \mathrm{EBF}=0.592 \mathrm{~W}+0.789 \mathrm{~T}+0.089 \\
& \text { Read } \mathrm{TBF}=0.592 \mathrm{~W}-0.789 \mathrm{~T}+0.089
\end{aligned}
$$

